\title{
A Model-Driven Approach to Estimate Atmospheric Visibility with Ordinary Cameras
}

\author{
Raouf Babari ${ }^{\mathrm{a}}$, Nicolas Hautière ${ }^{\mathrm{a}}$, Éric Dumont ${ }^{\mathrm{a}}$, Roland Brémond ${ }^{\mathrm{a}}$, Nicolas Paparoditis ${ }^{\mathrm{b}}$ \\ ${ }^{a}$ Université Paris-Est, LEPSIS, IFSTTAR, 58 boulevard Lefebvre, F-75015 Paris \\ ${ }^{b}$ Université Paris-Est, MATIS, IGN, 73 avenue de Paris, F-94160 Saint-Mandé
}

\begin{abstract}
Atmospheric visibility is an important input for road and air transportation safety, as well as a good proxy to estimate the air quality. A model-driven approach is presented to monitor the meteorological visibility distance through use of ordinary outdoor cameras. Unlike in previous data-driven approaches, a physics-based model is proposed which describes the mapping function between the contrast in the image and the atmospheric visibility. The model is non-linear, which allows encompassing a large spectrum of applications. The model assumes a continuous distribution of objects with respect to the distance in the scene and is estimated by a novel process. It is more robust to illumination variations by selecting the Lambertian surfaces in the scene. To evaluate the relevance of the approach, a publicly available database is used. When the model is fitted to short range data, the proposed method is shown to be effective and to improve on existing methods. In particular, it allows envisioning an easier deployment of these camera-based techniques on multiple observation sites.
\end{abstract}

Keywords: camera, visibility, observation, road safety, aviation safety, air quality

\section{Introduction}

In the presence of fog, haze or air pollution, atmospheric ${ }^{34}$ visibility is reduced. This constitutes a common and vexing ${ }^{35}$ transportation problem for different public authorities in multi- ${ }^{36}$ ple countries throughout the world.

First, low visibility is obviously a problem of traffic safety. ${ }^{38}$ Indeed, the behavior of drivers in fog is often inappropriate ${ }^{39}$ (e.g. reduced headways, altered reaction times) but the rea- ${ }^{40}$ sons for these dangerous behaviors are not fully understood ${ }^{41}$ (Kang et al., 2008; Caro et al., 2009). A recommendation in ${ }^{42}$ order to improve the safety in such situations was to use two ${ }^{43}$ rear fog lamps in the vehicles, as far apart as possible (Cavallo ${ }^{44}$ et al., 2001). It was also suggested that lowering the height of 45 these lamps could lead to reduced headway estimation (Buch- 46 ner et al., 2006). Various countermeasures have been tested on ${ }^{47}$ the roadside to reduce the impact of critically reduced visibil- 48 ity (Shepard, 1996), among which automated warning systems 49 employing road-side weather stations and visibility meters to ${ }^{50}$ provide automated detection (Mac Carley, 2005).

In addition to the road safety problem, reduced visibility is 52 cause of delays and disruption in air, sea and ground transporta- 53 tion for passengers and freight. On highways, massive pile- ${ }^{54}$ ups create non-recurrent congestions which sometimes force 55 the operator to momentarily close the road. Fog-related road ${ }^{56}$ closures are not an uncommon subject for news headlines. An- 57 other example is the Heathrow airport which was blocked for 58 three days during Christmas 2006. Such events have important 59 economic impacts (Pejovic et al., 2009). According to Perry 60 and Symons (1991), in 1974 fog was estimated to have cost 61 over roughly $£ 120$ millions (at 2010 prices) on the roads of 62 Great Britain. This figure includes the cost of medical treat- 63 ment, damage to vehicles and property, as well as the adminis- 64 trative costs of police, services and insurance, but they do not include the cost of delays to people not directly involved in the accident.

Impaired visibility is also a symptom of environmental problems because it is evidence of air pollution (Hyslop, 2009); in addition, it has been shown that impaired visibility in urban environment and mortality are correlated (Thach et al., 2010). According to Thach et al. (2010), visibility provides a useful proxy for the assessment of environmental health risks from ambient air pollutants, and a valid approach for the assessment of the public health impacts of air pollution where pollutant monitoring data are scarce.

The ability to accurately monitor visibility helps solving these problems. Critical safety at important transportation facilities such as airports are generally instrumented for monitoring visibility with devices that are expensive and hence, scarce. Cost is precisely the reason why highway meteorological stations are seldom equipped with visibility meters. In this context, using existing and ubiquitous highway cameras is of great interest, as these are low cost sensors already deployed for other purposes such as traffic monitoring (Jacobs et al., 2009). Furthermore, introducing new functionalities into roadside cameras would make them multipurpose and thus more cost-effective, easing their deployment along the roads.

Attempts at estimating the visibility using outdoor cameras or webcams are reported in the literature. However, the visibility range differs from one application to another, so that there is no general approach to tackle the problem by camera. For road safety applications, the range $0-400 \mathrm{~m}$ is usually considered. For meteorological observation and airport safety, the range 0$1000 \mathrm{~m}$ is usually considered. Visual range is also used for monitoring pollution in urban areas. In this case, higher visual 
ranges, typically $1-5 \mathrm{~km}$, are usually considered. In the follow-122 ing, we address the whole spectrum of visual ranges, typically ${ }_{123}$ $0-10 \mathrm{~km}$.

Two families of methods are proposed in the literature. The ${ }_{125}$ first one estimates the maximum distance at which a selected ${ }_{126}$ target can be detected. The methods differ depending on the ${ }_{127}$ nature of the target and how to estimate the distance. For in-128 telligent vehicles as well as for visual monitoring of highway ${ }_{129}$ traffic, a black target at the horizon is chosen and a flat road ${ }_{130}$ is assumed. Bush and Debes (1998) use a wavelet transform ${ }_{131}$ to detect the highest edge in the image with a contrast above ${ }_{132}$ $5 \%$. Based on a highway meteorology standard, Hautière et al.133 (2008) proposed a reference-free roadside camera-based sensor ${ }_{134}$ which not only estimates the visibility range but also estimates ${ }_{135}$ whether the visibility reduction is caused by fog. For meteoro-136 logical observations, regions of interest whose distance can be ${ }_{137}$ obtained on standard geographic maps are selected manually ${ }_{138}$ (Bäumer et al., 2008). An accurate geometric calibration of the ${ }_{139}$ camera with respect to the scene is necessary to calibrate and ${ }_{140}$ operate continuously these methods, which may be understood ${ }_{141}$ as direct approaches.

A second family of methods correlates the contrast in the scene with the visual range estimated by reference additional ${ }^{142}$ sensors (Hallowell et al., 2007). No accurate geometric calibration is needed. Conversely, a learning phase is needed to ${ }^{143}$ estimate the function which maps the contrast in the scene to the visual range. The method proposed in this paper belongs to this second family. Usually, a gradient based on the Sobel filter or a high-pass filter in the frequency domain are used to compute the contrast (Liaw et al., 2010; Hagiwara et al., 2007; Xie et al., 2008). Luo et al. (2005) have shown that the visual range obtained with these two approaches are highly correlated. Liaw et al. (2010) proposed to use a homomorphic filter or a Haar function in addition to the high-pass filter in order to reduce the effects of non-uniform illumination. Once the contrast is com- ${ }^{144}$ puted, a linear regression is performed to estimate the mapping ${ }^{145}$ function (Hallowell et al., 2007; Xie et al., 2008; Liaw et al., ${ }^{146}$ 2010). Babari et al. (2010) propose a method which is robust ${ }^{147}$ to illumination variations in the scene by taking into account ${ }^{148}$ the physical properties of objects in the scene. Unlike previous ${ }^{149}$ methods, a non-linear data regression is performed which allows covering a wider spectrum of applications. Due to the step of data regression, these methods can be seen as data-driven approaches. Nevertheless, the major problem of data-driven methods is the need of a learning phase, which makes this kind of method difficult to deploy massively. Indeed, one must wait for an episode with impaired visibility, so as to collect learning data and compute the fitting parameters.

The direct approaches are very sensitive to the geometric calibration of the camera but no learning phase is necessary to use them. The data-driven approaches do not use any accurate geometric calibration. However, they need episodes with impaired visibility before they are operational. We believe that new techniques can be developed which need neither accurate geometric calibration nor learning phase. In this aim, one must model how the contrast in the scene is altered by the presence of reduced visibility conditions, so as to build an a priori mapping func- tion between the contrast and the atmospheric visibility distance in the scene. This constitues a model-driven approach. Hautière et al. (2010) propose such a probabilistic model-driven approach which allows computing a physics-based mapping function. In particular, the model takes into account an a priori distribution of contrasts in the scene. However, a uniform distribution of targets is assumed which limits the applicability of the method on any scene. In this article, the method proposed in (Hautière et al., 2010) is generalized by adding new targets distributions, as well as a method to estimate the actual distribution of objects in the scene. A great attention is paid to the data fitting process, which greatly influences the final results. To assess the relevance of the approach, the different methods are compared using the MATILDA database (Hautière et al., 2010).

This article is organized as follows. In section 2, Koschmieder's model of fog visual effects is recalled. In section 3, the model-driven approach is presented, whose experimental evaluation is carried out in section 4. Finally, the results are discussed and perspectives for future work are given.

\section{Vision through the Atmosphere}

\subsection{Koschmieder's Theory}

The attenuation of luminance through the atmosphere was studied by Koschmieder (Middleton, 1952), who derived an equation relating the extinction coefficient of the atmosphere $\beta$, which is the sum of the scattering coefficient and of the absorption coefficient, the apparent luminance $L$ of an object located at distance $d$, and the luminance $L_{0}$ measured close to this object:

$$
L=L_{0} e^{-\beta d}+L_{\infty}\left(1-e^{-\beta d}\right)
$$

(1) indicates that the luminance of the object seen through fog is attenuated by $e^{-\beta d}$ (Beer-Lambert law); it also reveals a luminance reinforcement of the form $L_{\infty}\left(1-e^{-\beta d}\right)$ resulting from daylight scattered by the slab of fog between the object and the observer, the so-called airlight. $L_{\infty}$ is the atmospheric luminance.

On the basis of this equation, Duntley developed a contrast attenuation law (Middleton, 1952), stating that a nearby object exhibiting contrast $C_{0}$ with the fog in the background will be perceived at distance $d$ with the following contrast:

$$
C=\left[\frac{L_{0}-L_{\infty}}{L_{\infty}}\right] e^{-\beta d}=C_{0} e^{-\beta d}
$$

This expression serves to base the definition of a standard dimension called meteorological visibility distance $V$, i.e. the greatest distance at which a black object $\left(C_{0}=-1\right)$ of a suitable dimension can be seen on the horizon, with the threshold contrast set at 5\% (CIE, 1987). It is thus a standard parameter that characterizes the opacity of a fog layer. This definition yields the following expression:

$$
V \approx \frac{3}{\beta}
$$




\subsection{Contrast of Lambertian Targets}

Assuming a linear response function of the camera, the in-173 tensity $I$ of a distant point located at distance $d$ in an outdoor scene is given by Koschmieder's model (1):

$$
I=R e^{-\beta d}+A_{\infty}\left(1-e^{-\beta d}\right)
$$

where $R$ is the intrinsic intensity of the pixel, i.e. the inten- ${ }^{177}$ sity corresponding to the intrinsic luminance value of the cor- ${ }^{-178}$ responding scene point and $A_{\infty}$ is the background sky intensity. ${ }^{179}$ Two points located at roughly the same distance $d_{1} \approx d_{2}=d$ with different intensities $I_{1} \neq I_{2}$ form a distant target whose normalized contrast is given by:

$$
C=\frac{I_{2}-I_{1}}{A_{\infty}}=\left[\frac{R_{2}-R_{1}}{A_{\infty}}\right] e^{-\beta d}=C_{0} e^{-\beta d}
$$

In this equation, the contrast $C$ of a target located at distance ${ }^{183}$ $d$ depends on $V=\frac{3}{\beta}$ and on its intrinsic contrast $C_{0}$. If $\mathrm{we}^{184}$ now assume that the surface of the target is Lambertian, the ${ }^{185}$ luminance $L$ at each point $i$ of the target is given by:

$$
L=\rho_{i} \frac{E}{\pi}
$$

where $E$ denotes the global illumination and $\rho_{i}$ denotes the albedo at $i$. Moreover, it is a classical assumption to set $L_{\infty}=\frac{E}{\pi}$ so that (5) finally becomes:

$$
C=\left(\rho_{2}-\rho_{1}\right) e^{-\beta d} \approx\left(\rho_{2}-\rho_{1}\right) e^{-\frac{3 d}{V}}=\Delta \rho e^{-\frac{3 d}{V}}
$$

Consequently, the contrast of a distant Lambertian target only depends on its physical properties and on its distance to the sensor and on the meteorological visibility distance, and no longer on the illumination. These surfaces are robust to strong illumination variations in the computation of the contrast in the scene.

\section{The Model-Driven Approach}

\subsection{Principle}

Let us consider an outdoor scene where targets are distributed 190 continuously at increasing distances from the camera. Let us denote $\phi$ the probability density function of observing a contrast $C$ in the scene:

$$
\mathbb{P}(C<X \leq C+\mathrm{d} C)=\phi(C) \mathrm{d} C
$$

The expectation of the contrast $m$ in the image is expressed as:

$$
m=\mathbb{E}[C]=\int_{0}^{1} C \phi(C) \mathrm{d} C
$$

Based on (7), $C$ is a random variable which depends of the two random variables $d$ and $\Delta \rho$. These two variables are assumed to be independent, which allows expressing (9) as:

$$
m=\mathbb{E}[\Delta \rho] \mathbb{E}\left[e^{-\frac{3 d}{V}}\right]=\overline{\Delta \rho} \int_{0}^{+\infty} \psi(d) e^{-\frac{3 d}{V}} \mathrm{~d} d
$$

where $\overline{\Delta \rho}$ denotes the mean albedo difference between the objects in the scene and $\psi$ denotes the p.d.f. of there being an object at the distance $d$ in the scene. To compute $m$, a realistic expression for the density of objects $\psi$ in the scene is needed.

\subsection{Expectation of the Contrast}

Choosing a suitable target distribution $\psi$ allows us computing the expectation of the contrast (10) with respect to the meteorological visibility distance. In (Hautière et al., 2010), (10) was solved assuming a uniform distribution of targets between 0 and $d_{\max }$, which leads to the following solution:

$$
m_{u}=\frac{V \overline{\Delta \rho}}{3 d_{\max }}\left[1-\exp \left(-\frac{3 d_{\max }}{V}\right)\right]
$$

This assumption may be useful when the scene is not known a priori but may limit the applicability of the method on any scene. The problem has received little consideration in the literature. Torralba and Oliva (2002) proposed some a priori depth distributions in natural or man-made scenes which are Gaussian distributions. To circumvent this problem, a solution is to estimate the actual distribution and to solve $m$ for this distribution. Let us first examine if mathematical solutions exist for classical statistical distributions.

Assuming a Gaussian distribution of parameters $\mu$ and $\sigma$, the density of targets is given by:

$$
\psi_{G}(d)=\frac{1}{\sigma \sqrt{2 \pi}} \exp \left[-\frac{1}{2}\left(\frac{d-\mu}{\sigma}\right)^{2}\right]
$$

(10) then has an analytical solution $m_{g}$,which is given by:

$$
m_{G}(V)=\frac{\overline{\Delta \rho}}{2} \exp \left(\frac{9 \sigma^{2}}{2 V^{2}}-\frac{3 \mu}{V}\right) \operatorname{erfc}\left[\frac{1}{\sigma \sqrt{2}}\left(\frac{3 \sigma^{2}}{V}-\mu\right)\right]
$$

where erfc denotes the complementary error function:

$$
\operatorname{erfc}(z)=\frac{2}{\sqrt{\pi}} \int_{z}^{\infty} \exp \left(-\zeta^{2}\right) \mathrm{d} \zeta
$$

In the same way, assuming a Rayleigh distribution of parameter $\sigma$ :

$$
\begin{aligned}
& \psi_{R}(d)=\frac{d}{\sigma^{2}} \exp \left(\frac{-d^{2}}{2 \sigma^{2}}\right) \\
& m_{R}(V)=1-\frac{3 \sigma \overline{\Delta \rho}}{V} \exp \left(\frac{9 \sigma^{2}}{2 V^{2}}\right) \sqrt{\frac{\pi}{2}} \operatorname{erfc}\left(\frac{3 \sigma}{V \sqrt{2}}\right)
\end{aligned}
$$




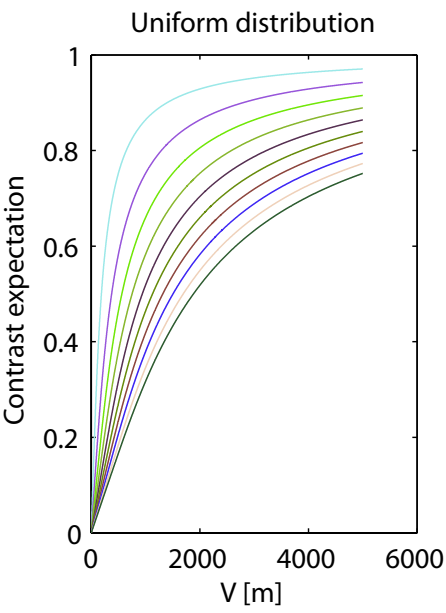

(a)

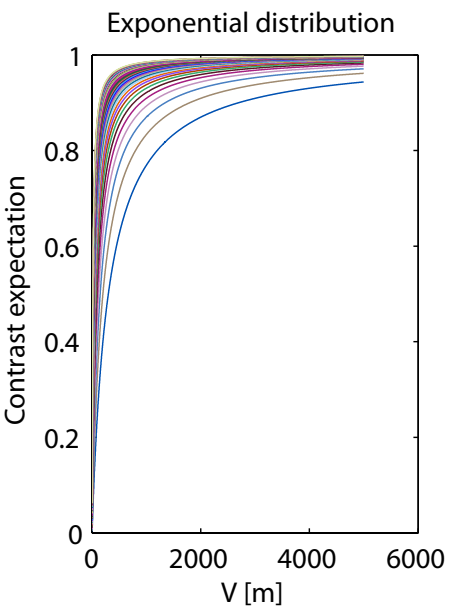

(b)

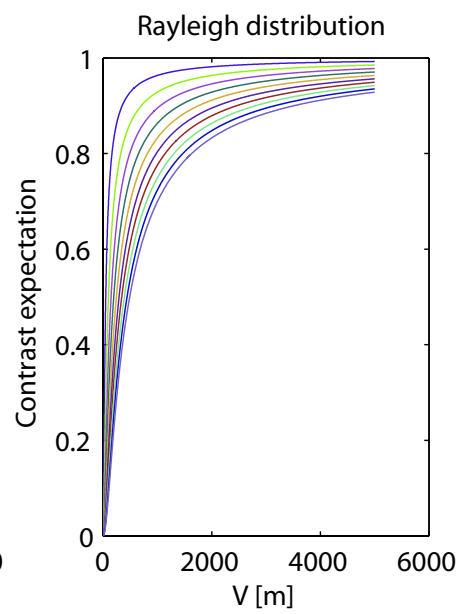

(c)

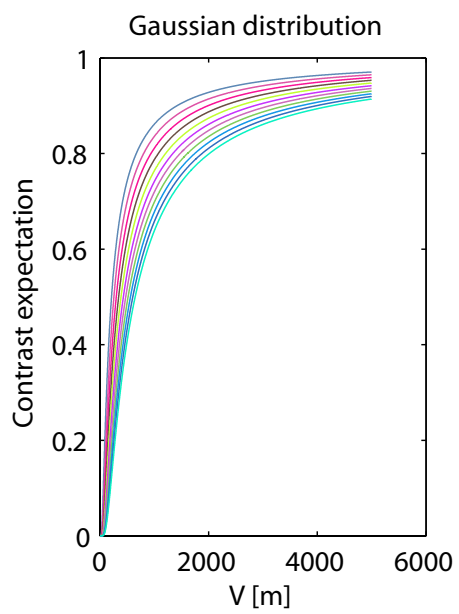

(d)

Figure 1: Plots of the different contrast expectation models assuming (a) a uniform distribution $\left(d_{\max } \in[100 ; 1000]\right)$; (b) an exponential distribution of targets density $(v \in[0.01 ; 0.1]) ;(\mathrm{c})$ a Rayleigh distribution of targets density $(\sigma \in[10 ; 100]) ;(\mathrm{d})$ a Gaussian distribution of targets density $(\sigma=10$ and $\mu \in[50 ; 150])$.

Finally, assuming an exponential distribution of parameter $v$ :

$$
\begin{aligned}
\psi_{e}(d) & =v \exp (-v d) \\
m_{e}(V) & =\frac{v \overline{\Delta \rho}}{v+\frac{3}{V}}
\end{aligned}
$$

Other types of distributions can be tested, such as the lognormal distribution. However, mathematical solutions are not easy to find and then to handle, apart from the uniform and exponential distributions.

\subsection{Model Inversion and Error Estimation}

The different models are all increasing functions of $V$ and share the same limits towards 0 and $\infty$, see Eqs. $(11,13,16,18)$ :

$$
\lim _{V \longrightarrow 0} m=0 \quad \lim _{V \longrightarrow \infty} m=1
$$

which are obvious physical bounds that data-driven approaches do not always respect. The models of contrast expectation presented in the previous section are plotted as functions of the meteorological visibility distance $V$ in Fig. 1. As one can see,202 these models have roughly the same shape.

In (Hautière et al., 2010), the solution for the uniform case ${ }_{20}$ was found to be invertible:

$$
V\left(m_{u}\right)=\frac{3 m_{u} d_{\max }}{1+m_{u} W\left(\frac{e^{-1 / m_{u}}}{m_{u}}\right)}
$$

where $W$ denotes the Lambert function, which is a transcendental function defined by solutions of the equation $W(x) e^{W(x)}=x$ (Corless et al., 1996). Given the complexity of the equation, it is somehow difficult to compute the partial derivatives of the model and express error bounds of the model. In the case of the Gaussian and Rayleigh distributions, it is also possible to find analytical solutions to invert the models, but these ones205 are not detailed here for the sake of readability of the article. 206

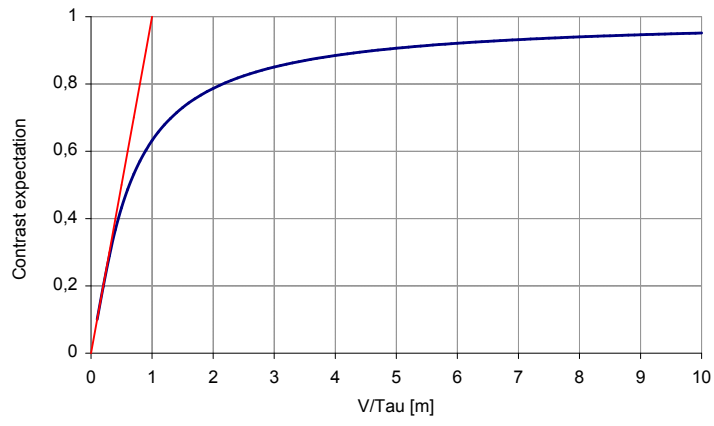

Figure 2: Analogy between the charge/discharge of a capacitor and the shape of the contrast expectation (blue curve) with respect to the meteorological visibility. The red curve denotes the tangent at the origin.

Fortunately in the case of an exponential distribution, a simpler solution is available:

$$
V\left(m_{e}, v\right)=\frac{3 m_{e}}{v\left(1-m_{e}\right)}
$$

With this model, the partial derivatives of $V$ with respect to $m$ and $v$ (22) can be obtained and an upper bound of the error of the model (23) is derived:

$$
\begin{aligned}
\mathrm{d} V & =\frac{\partial V\left(m_{e}, v\right)}{\partial m_{e}} \mathrm{~d} m_{e}+\frac{\partial V\left(m_{e}, v\right)}{\partial v} \mathrm{~d} v \\
\Delta V & \leq \frac{3 \Delta m_{e}}{v\left(1-m_{e}\right)^{2}}+\frac{3 m_{e} \Delta v}{v^{2}\left(1-m_{e}\right)}
\end{aligned}
$$

At this stage, we can make a comparison with the charging/discharging of a capacitor. Assuming a uniform distribution, (11) can be expressed as following:

$$
m_{u}=\overline{\Delta \rho} \frac{V}{\tau}\left[1-\exp \left(-\frac{\tau}{V}\right)\right]
$$

where $\tau=3 d_{\max }$. When $V=\tau$, we have $m_{u}=1-e^{-1} \approx 0.63$. This is the same constant as the one used to characterize the 
charging speed of a capacitor. Fig. 2 shows the curve obtained when plotting (24) with respect to the ratio $\frac{V}{\tau}$.

In the general case, the capacitance of the system is determined by the distribution of distances in the scene, the texture of the objects in the scene and the quality (MTF, resolution) of the camera along with the response of the image processing filter (e.g. the Sobel filter). The smaller the capacitance of the system, the faster the curves go to 1 . We thus define an indicator $\tau$ of the system quality which is the meteorological visibility distance at which 0.63 of the "capacitance" is reached.

\subsection{Estimation of the Distribution of Targets}

The direct computation of $m$ and $V$ strongly depends on the distribution $\psi$. Thus, an important task is to guess which distribution is best suited for a given scene. Following the method proposed by Narasimhan and Nayar (2003), the scene structure can be approximated from two weather conditions 1 and 2 thanks to Koschmieder's law (1):

$$
\left(\beta_{2}-\beta_{1}\right) d=-\log \left[\frac{A_{\infty_{2}}-I_{2}}{A_{\infty_{1}}-I_{1}}\right]-\log \frac{A_{\infty_{1}}}{A_{\infty_{2}}}
$$

Using this method, it is possible to roughly estimate a depth for each pixel of the scene image. Starting from Narasimhan and Nayar (2003), we used landmarks of known depth and we adjusted the sky intensities $A_{\infty_{1}}$ and $A_{\infty_{2}}$ so as to improve the accuracy of the global map.

Second, due to the noise of the camera sensor, a simple computation of the depth distribution is useless. Soft-voting is often used to obtain reliable data from multiple uncertain data sources ${ }^{242}$ (Latif-Shabgahi et al., 2004). In computer vision and pattern ${ }^{243}$ recognition, this process is often used to deduce a global infor- ${ }^{244}$ mation from local information, e.g. the Hough transform (Duda ${ }^{245}$ and Hart, 1972), the fast radial symmetry transform (Loy and ${ }^{246}$ Zelinsky, 2003) or the v-disparity transform (Labayrade et al., ${ }^{24}$ 2002). In a similar way, the distribution of Lambertian targets can be estimated using a Parzen's like approach (Parzen, 1962).248

In this aim, a cumulative histogram of depth $h(d)$ is computed for $d \in\left[0, d_{\max }\right]$ which takes into account a bandwidth ${ }^{249}$ parameter. This one is related to the confidence $u_{i}$ on the esti-250 mation of the distance associated to each pixel. For each pixel, ${ }^{251}$ a normal distribution $\mathscr{N}\left(d \mid d_{i}, u_{i}\right)$ is cumulated in the histogram ${ }^{252}$ with center $d_{i}$ and standard deviation $u_{i}$. In addition to the ${ }^{253}$ standard Parzen's approach, we also use a weighting parame-254 ter $w_{i}$ which accounts for the contribution of each data to the histogram. This histogram of depth is then expressed by: ${ }^{255}$

$$
h(d)=\sum_{i=1}^{P} w_{i} \mathscr{N}_{i}\left(d \mid d_{i}, u_{i}\right)
$$

where $P$ denotes the total number of pixels. The confidence $u$ is ${ }^{259}$ obtained by computing the sensitivity of (25) to its parameters: ${ }^{260}$

$$
u \propto \sum\left[\frac{\partial d}{\partial\left(A_{\infty_{1,2}}, I_{1,2}\right)} \mathrm{d}\left(A_{\infty_{1,2}}, I_{1,2}\right)\right]^{2}
$$

Assuming $\mathrm{d} A_{\infty_{1}} \approx \mathrm{d} A_{\infty_{2}} \approx \mathrm{d} I_{1} \approx \mathrm{d} I_{2}=\mathrm{d} I$, (27) becomes:

$$
u \propto \frac{f_{1}+f_{2}}{\left(\beta_{2}-\beta_{1}\right)^{2}} \mathrm{~d} I^{2}
$$

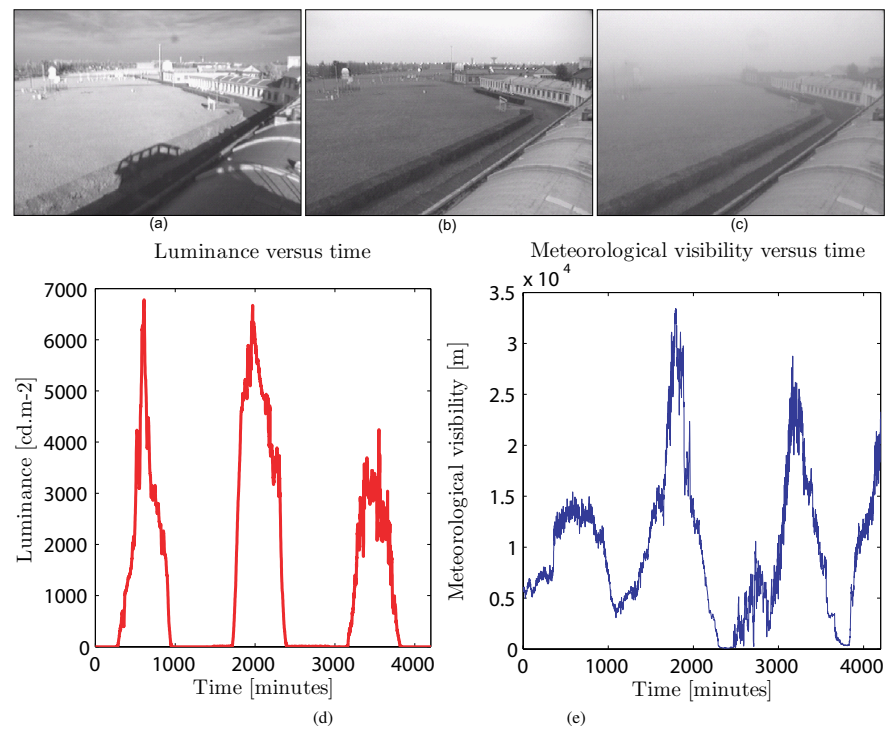

Figure 3: Samples of data collected in winter 2008-2009: (a) images with strong illumination conditions and presence of shadows; (b) cloudy conditions; (c) foggy weather situation; (d) meteorological visibility distance data and (e) background luminance data collected in the field test during two days.

where $f_{i}$ is given by:

$$
f_{i=1,2}=\frac{1}{A_{\infty_{i}}{ }^{2}}+2\left[\frac{1}{\left(A_{\infty_{i}}-I_{i}\right)^{2}}+\frac{1}{A_{\infty_{i}}\left(A_{\infty_{i}}-I_{i}\right)}\right]
$$

In section 4.4, we apply this method to actual data issued from a test site. In particular, we chose the relevant weight $w_{i}$. Having estimated $h$, the relevant distribution model can be determined empirically or using classical statistical tests. If the distribution has different modes, a probability mixture model can also be used to fit $h$.

\section{Experimental Validation}

In this section, an experimental evaluation of the proposed approach for visibility estimation is carried out. In this aim, the publicly available MATILDA database is used. First, the methodology is presented. Second, a method to estimate wether a surface is Lambertian or not is recalled. Third, results are presented and discussed.

\subsection{Experimental Data}

The observation test field is equipped with a reference transmissometer (Degreane Horizon TI8510). It serves to calibrate different scatterometers (Degreane Horizon DF320) used to monitor the meteorological visibility distance on the French territory, one of which provided our data. They are coupled with a background luminance sensor (Degreane Horizon LU320) which monitors the illumination received by the sensor. A camera grabs images of the field test every ten minutes. This camera is an 8-bit CCD camera $(640 \times 480$ definition, mounting height $8.3 \mathrm{~m}$, pitch angle $9.8^{\circ}$, focal length $f_{l}=4 \mathrm{~mm}$ and pixel size $\left.t_{p i x}=9 \mu \mathrm{m}\right)$. It is thus a low cost camera which is representative of common video surveillance cameras. 


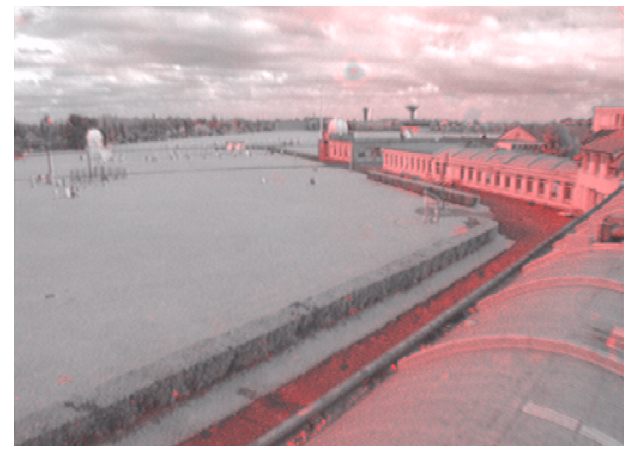

Figure 4: Map of Lambertian surfaces on the field test: The redder the pixel is, the higher the probability that the surface is Lambertian.

\subsection{Contrast Estimator}

Having located the Lambertian surfaces, the gradients in the scene are estimated by means of the module of the Sobel filter. ${ }^{316}$ For each pixel, the gradient $\nabla_{i, j}$ is normalized by the intensity ${ }_{317}$ of the background $A_{\infty}$. Since the camera is equipped with an $_{318}$ auto gain control, the background intensity $A_{\infty}$ is most of the $e_{319}$ time equal to $2^{8}-1$, so that this step can be skipped. Each gra-з20 dient is then weighted by $P_{i, j}^{L}$, the probability that a pixel $(i, j)_{321}$ belongs to a Lambertian surface. Consequently, only relevant 322 areas of the image are used for the visibility estimation, and $_{323}$

Two fog events were collected at the end of February 2009.305 The fog occurred early in the morning and lasted a few 30 The meteorological visibility distances and luminances areso plotted in Figs. 3(d)\&(e) versus time on a three day pe-311 iod. As one can see, the meteorological visibility distance ${ }_{312}$ ges from $100 \mathrm{~m}$ to $35,000 \mathrm{~m}$ and the luminance ranges ${ }_{31}$ $6,000 \mathrm{~cd} \cdot \mathrm{m}^{-2}$. This database made of 150 images 3 site http://www.lcpc.fr/en/produits/matilda/ for research purpose.

uted on the normalized gradient is comsection 3. Locating the Lambertian surfaces in the images is hus needed. Following the method proposed in Babari et al. , the Pearson coefficient, denoted $P_{i, j}^{L}$, is computed beof the sun changes and the value of the background luminance estimated by the luminancemeter. The closer $P_{i, j}^{L}$ is to 1 , the tronger the probability that the pixel belongs to a Lambertian This technique provides an efficient way to locate the the scene. For the MATILDA database, redder the pixel, the higher the probability that the surface is Lambertian.

the scene need not be totally Lambertian. Finally, the estimated contrast in the scene $\tilde{m}$ is given by:

$$
\tilde{m}=\frac{1}{N} \sum_{i, j} \Delta \rho_{i, j} \exp \left(-\frac{3 d_{i, j}}{V}\right) P_{i, j}^{L}=\frac{1}{N} \sum_{i, j} \frac{\nabla_{i, j}}{A_{\infty}}
$$

where $\Delta \rho_{i, j}$ is the intrinsic contrast of a pixel (7) and $N$ denotes the number of pixels of the image.

\subsection{Selection of the Relevant Distribution}

In section 3.4 , we have proposed a methodology to estimate the distribution $\psi$ in a scene. In this section, we apply this method to the test site of the MATILDA database. Having the contrast estimator (see previous paragraph), we are now able to derive a relevant weight $w_{i}$. Based on (30), the contribution of a data to the histogram is its weighted gradient $\nabla_{i, j} P_{i, j}^{L}$ computed in good weather conditions, which leads to choose it as weight $w_{i}$, see (26). The confidence $u_{i}$ on the depth of each pixel is given by (28) and it is controlled by the value of $\mathrm{d} I$ which is set empirically. The estimated distribution is shown in Fig. 5 using the green plot $(\mathrm{d} I=0.1)$, the purple plot $(\mathrm{d} I=0.25)$ and the black curve $(\mathrm{d} I=1)$. The exponential distribution fits the data quite well and is chosen to model the data of the histogram because it is the most easily revertible and is plotted in red. Based on this curve, we estimate $d_{\max } \approx 325 \mathrm{~m}$. We can thus expect a capacitance $\tau$ of approximately $1000 \mathrm{~m}$.

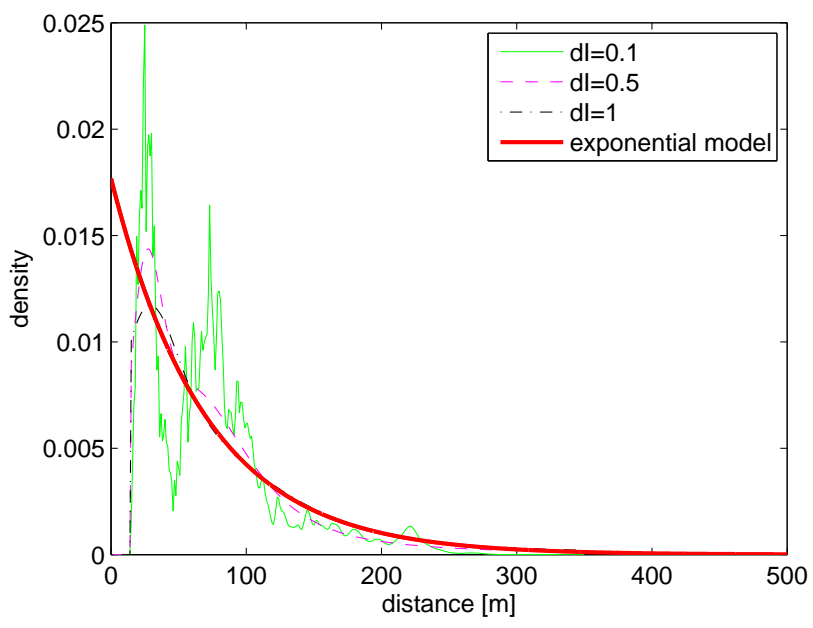

Figure 5: Histogram of weighted contrasts versus depth. The estimated distribution is shown using the green plot $(\mathrm{d} I=0.1)$, the purple plot $(\mathrm{d} I=0.5)$ and the black curve $(\mathrm{d} I=1)$. The fitted exponential distribution is plotted in red.

\subsection{Results}

As in Babari et al. (2010), $\tilde{m}$ is computed for the collection of 150 images of the MATILDA database using (30). The exponential distribution model (18) has been fitted to all the data using a robust non-linear least squares fitting technique $\left(R^{2}=\right.$ 0.91), namely the Levenberg-Marquardt algorithm. We have also fitted upper and lower bound curves which comprise $99 \%$ of the data points. The different curves are plotted in Fig. 6(a). 


\begin{tabular}{|c|c|c|c|c|c|}
\hline Application & Highway fog & Meteorological fog & Haze & \multicolumn{2}{|c|}{ Air quality } \\
\hline Range [m] & $0-400$ & $0-1000$ & $0-5000$ & $0-10000$ & $0-15000$ \\
\hline Number of data & 13 & 19 & 45 & 70 & 150 \\
\hline Weighted logarithmic model (Babari et al., 2010) & $10.4 \%$ & $22.5 \%$ & $23.4 \%$ & $29.9 \%$ & $41.9 \%$ \\
Uniform distribution (Hautière et al., 2010) & $12.6 \%$ & $18.1 \%$ & $29.7 \%$ & $\infty$ & $\infty$ \\
Exponential distribution & $10.0 \%$ & $16.2 \%$ & $29 \%$ & $60 \%$ & $373 \%$ \\
Exponential distribution + enhanced fitting & $9.7 \%$ & $11.2 \%$ & $33 \%$ & $50 \%$ & $63.5 \%$ \\
\hline
\end{tabular}

Table 1: Mean relative errors of meteorological visibility distance estimation with respect to the envisioned applications.
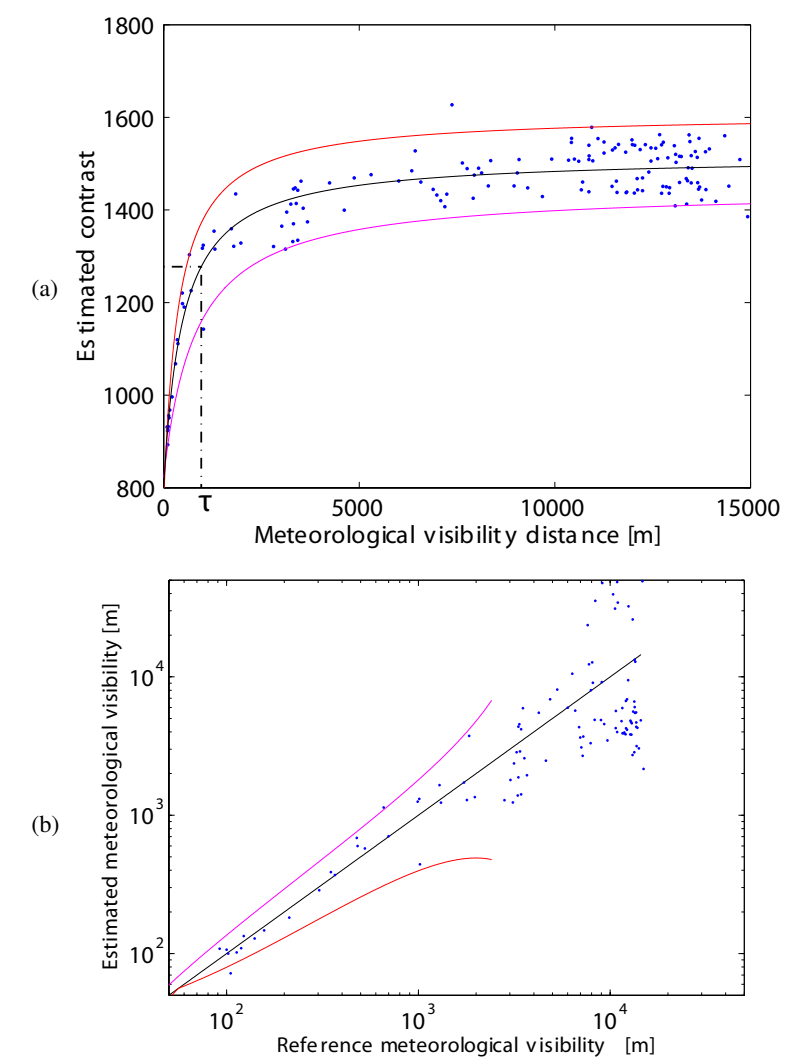

Figure 6: Model fitting: (a) Data fitting with the exponential distribution model ${ }^{365}$ in black. The upper bound is plotted in blue and the lower bound in magenta.366 (b) Plot of estimated visibility distances versus reference visibility distances.
We estimated a capacitance of the scene $\tau \approx 950 \mathrm{~m} \approx 3 d_{\max }$ as ${ }^{369}$ expected. We invert the fitted model using (21) and estimate the ${ }^{370}$ meteorological visibility distance based on the contrast expec- ${ }^{37}$ tation $m$. Finally, we plot the estimated meteorological visibil- ${ }_{373}$ ity distance versus the reference meteorological visibility distance in Fig. 6(b). From the same experimental data, Babari et al. (2010) fit an empirical logarithmic model, whereas Hautière et al. (2010) fit the contrast expectation of a uniform dis- ${ }_{375}$ tribution (11).

The mean relative errors are compared in Tab. 1. Since the $e_{376}$ applications are very different depending on the range of me-377 teorological visibility distances, the relative error for various 378 applications are computed: road safety, meteorological obser-379 vation and air quality.
Compared to data-driven approaches, one can see that the error remains low with model-driven approaches for critical safety applications, increases for higher visibility ranges, and becomes huge for visibility distances above $7 \mathrm{~km}$. On the test site, using the actual target distribution, i.e. the exponentiel model, improves the previous results obtained with the uniform distribution (Hautière et al., 2010) and covers a large spectrum of applications with a limited error. Due to the unbalanced data fitting process, the error is slightly higher for low visibility ranges $(<1.000 \mathrm{~m})$ using the data-driven approach (Babari et al., 2010) despite the weighting introduced by the authors. It remains limited for higher visibility ranges $(>5000 \mathrm{~m})$.

In the previous results, all the data have been used to fit the models. This is the principle underlying the data-driven approach. Conversely, this approach should not be followed for the model-driven approach, since the model may not be valid for the whole ranges of visibility. According to section 4.4, we are sure that the model is valid in the range $0-\tau$, i.e. $0-1000$ $\mathrm{m}$ in our case. A new data fitting process is deduced. First, the exponential distribution model (18) has been fitted to the data in the range 0-1000 $\mathrm{m}$ using a robust non-linear least squares fitting technique, namely the Levenberg-Marquardt algorithm. The confidence in the fitting is higher $\left(R^{2}=0.97\right)$. The fitted curve is shown in Fig. 7. Second, the model is extrapolated on the range $\tau-15000 \mathrm{~m}$. The mean relative error is then computed between the adjusted model and the ground truth data. The results are given in the last line of Tab. 1. Since the model has been fitted to short visibility data, the results are improved at short ranges. At higher ranges, the errors are reduced as well, which illustrates the benefits of performing a data fitting process only on reliable data.

Finally, according to metrology practices in the field of visibility observations, a measurement device is considered as correct if the error is smaller than $20 \%$ in $90 \%$ of the cases. The $10 \%$ worst cases are thus excluded from the error computation. In this way, we are able to obtain a correct estimate of the meteorological visibility up to $3320 \mathrm{~m}$.

\section{Discussion}

The data-driven approach requires visibility data for its calibration and implementation. Both model-driven approaches need only to determine the type of targets distribution in the scene. The distributions used in this article, namely uniform and exponential, are parameterized by a single parameter $d_{\max }$ 


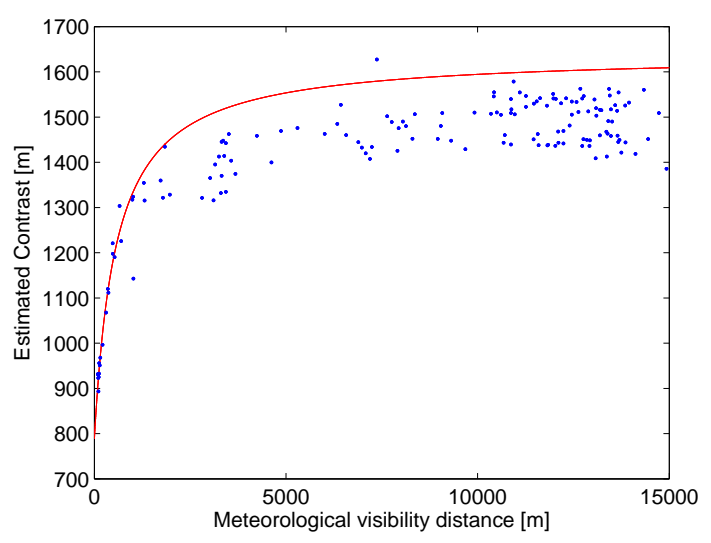

Figure 7: Enhanced data fitting process with the exponential distribution model ${ }^{430}$ on short visibility data and extrapolated on higher visibility ranges. The data431 are plotted in blue. The fitted model is plotted in red.

and can be guessed using only two images grabbed in different ${ }^{434}$ foggy weather conditions. Visibility data are thus no longer re- ${ }^{435}$ quired, which is an important progress towards generic methods without any learning phase.

However, a continuous distribution of object with respect to the distance in the scene is assumed. This assumption may be ${ }_{440}^{439}$ false in urban areas, where depth discontinuities exist because ${ }^{440}$ of the presence of vertical buildings. Using the actual depth ${ }_{442}^{44}$ distribution of the scene could improve the results. In this aim, spatial information systems could be used to estimate a more accurate depth distribution at a camera location, so as to get rid of the process proposed in section 3.4. However, it may be ${ }^{445}$ difficult to register accurately the 3-D GIS on the image.

Another limitation observed in the test scene is due to the fact that the range of distribution of Lambertian targets is $\mathrm{a}^{447}$ few hundred meters. We are thus not able to use all the visual cues which are present in the landscape. This can be due ${ }^{448}$ to non-uniform illumination when selecting Lambertian targets ${ }^{449}$ and could be reduced using the image processing filter proposed ${ }^{450}$ by Liaw et al. (2010). A second solution consists in changing the location of the camera and for example increasing its451 mounting height, so as to get a better perspective. A complementary solution consists in using a camera of better quality, $\mathrm{s}_{453}{ }_{452}$ as to get less noisy images. We aim at exploring these different 454 strategies.

Nevertheless, thanks to results shown in this article, we be- ${ }^{456}$ lieve that an ordinary camera is able to monitor the atmospheric ${ }_{458}^{47}$ visibility whatever the envisaged application: road safety, aero-459 nautic and air quality. This allows envisioning the development $t^{460}$ of multipurpose environmental monitoring cameras.

\section{Conclusion}

Camera-based methods are being developed to estimate the ${ }^{468}$ atmospheric visibility. However, the methods are either dedi- ${ }^{469}$ cated to road safety (low visibility ranges) or to air quality mon- ${ }_{471}^{470}$ itoring (high visibility ranges).
In this article, a generic model-driven approach is presented, which estimates the atmospheric visibility distance through use of ordinary outdoor cameras based on the contrast expectation in the scene. Unlike previous data-driven approaches, a physics-based model is proposed which expresses the mapping between the contrast and the atmospheric visibility distance. Contrary to previous approaches, the model is non-linear which explains why it is able to encompass a larger spectrum of applications. Due to its intrinsic physical constraints, the calibration of the system is also less sensitive to the input data. In particular, the model takes into account the actual distribution of visual targets in the scene, which is estimated by a novel dedicated process which only needs two different fog images. Visibility data are thus not mandatory anymore to calibrate the system. It is also invariant to illumination variations in the scene by selecting the Lambertian surfaces in the scene.

To evaluate the relevance of our approach, the publicly available MALTILDA database is used. Using these experimental data, promising results are obtained, which improve the previous the results obtained with this database. When models are fitted to all data, data-driven approaches seem to be more effective for high visibility ranges. When the non-linear models are fitted to the reliable data only, the data-driven approach and the model-driven approach give more or less the same results.

In future work, an ambitious objective is to estimate the contrast expectation function without any additional meteorological sensor, based only on the characteristics of the camera and the properties of the scene (geometry, texture) collected by remote sensing techniques. Such a generic model-driven approach would pave the road to methods without any constraining learning phase.

\section{Acknowledgments}

The work presented in this paper is co-funded by the IFSTTAR and Météo-France. The authors wish to thank IGN for his contribution to the supervision of this work.

\section{References}

Babari, R., Hautière, N., Dumont, E., Paparoditis, N., Misener, J., 2010. Visibility monitoring using conventional roadside cameras: Shedding light on and solving a multi-national road safety problem. Transportation Research Board Annual Meeting Compendium of Papers (TRB'11), Washington, D.C., USA .

Buchner, A., Brandt, M., Bell, R., Weise, J., 2006. Car backlight position and fog density bias observer-car distance estimates and time-to-collision judgments. Human Factors: The Journal of the Human Factors and Ergonomics Society 48, 300-317.

Bäumer, D., Versick, S., Vogel, B., 2008. Determination of the visibility using a digital panorama camera. Atmospheric Environment 42, 2593-2602.

Bush, C., Debes, E., 1998. Wavelet transform for analyzing fog visibility. IEEE Intelligent Systems 13, 66-71.

Caro, S., Cavallo, V., Marendaz, C., Boer, E.R., Vienne, F., 2009. Can headway reduction in fog be explained by impaired perception of relative motion? Human Factors, Human Factors: The Journal of the Human Factors and Ergonomics Society 51, 378-392.

Cavallo, V., Colomb, M., Doré, J., 2001. Distance perception of vehicle rear lights in fog. Human Factors: The Journal of the Human Factors and Ergonomics Society 43, 442-451

CIE, 1987. International Lighting Vocabulary. 17.4. 
Corless, R.M., Gonnet, G.H., Hare, D.E.G., Jeffrey, D.J., Knuth, D.E., 1996.54 On the Lambert W function. Advances in Computational Mathematics 5,545 329-359.

Duda, R.O., Hart, P.E., 1972. Use of the hough transformation to detect lines547 and curves in pictures. Communications of the ACM 15, 11-15.

Hagiwara, T., Ota, Y., Kaneda, Y., Nagata, Y., Araki, K., 2007. A method of 549 processing CCTV digital images for poor visibility identification. Trans-550 portation Research Records: Journal of the Transportation Research Board551 1973, 95-104

Hallowell, R., Matthews, M., Pisano, P., 2007. An automated visibility detec-553 tion algorithm utilizing camera imagery, in: 23rd Conference on Interactive Information and Processing Systems for Meteorology, Oceanography, and Hydrology (IIPS), San Antonio, TX, Amer. Meteor. Soc.

Hautière, N., Bigorgne, E., Bossu, J., Aubert, D., 2008. Meteorological conditions processing for vision-based traffic monitoring, in: International Workshop on Visual Surveillance, European Conference on Computer Vision.

Hautière, N., Babari, R., Dumont, E., Brémond, R., Paparoditis, N., 2010. Estimating meteorological visibility using cameras: A probabilistic modeldriven approach, in: Asian Conference on Computer Vision.

Hautière, N., Tarel, J.P., Aubert, D., 2007. Towards fog-free in-vehicle vision systems through contrast restoration, in: IEEE Conference on Computer Vision and Pattern Recognition, Minneapolis, Minnesota, USA.

He, K., Sun, J., Tang, X., 2009. Single image haze removal using dark channel prior, in: IEEE Conference on Computer Vision and Pattern Recognition, Miami, Florida, USA.

Hyslop, N.P., 2009. Impaired visibility: the air pollution people see. Atmospheric Environment, 43, 182-195.

Jacobs, N., W., B., Fridrich, N., Abrams, A., Miskell, K., Brswell, B., Richardson, A., Pless, R., 2009. The global network of outdoor webcams: Properties and apllications, in: ACM International Conference on Advances in Geographic Information Systems.

Kang, J., Ni, R., Andersen, G.J., 2008. Effects of reduced visibility from fog on car-following performance. Transportation Research Record: Journal of the Transportation Research Board , 9-15.

Labayrade, R., Aubert, D., Tarel, J.P., 2002. Real time obstacle detection in stereovision on non flat road geometry through v-disparity representation, in: IEEE Intelligent Vehicles Symposium.

Latif-Shabgahi, G., Bass, J.M., Bennett, S., 2004. A taxonomy for software voting algorithms used in safety-critical systems. IEEE Transactions on Reliability 53, $319-328$

Liaw, J.J., Lian, S.B., Huang, Y.F., Chen, R.C., 2010. Using sharpness image with haar function for urban atmospheric visibility measurement. Aerosol and Air Quality Research 10, 323-330.

Loy, G., Zelinsky, A., 2003. Fast radial symmetry for detecting points of interest. IEEE Transactions on Pattern Analysis and Machine Intelligence 25, 959-973.

Luo, C.H., Wen, C.Y., Yuan, C.S., Liaw, J.-L. ans Lo, C.C., Chiu, S.H., 2005. Investigation of urban atmospheric visibility by high-frequency extraction: Model development and field test. Atmospheric Environment 39, 2545 2552.

Mac Carley, C.A., 2005. Methods and metrics for evaluation of an automated real-time driver warning system. Transportation Research Record: Journal of the Transportation Research Board, 87-95.

Middleton, W., 1952. Vision through the atmosphere. University of Toronto Press.

Narasimhan, S.G., Nayar, S.K., 2003. Contrast restoration of weather degraded images. IEEE Transactions on Pattern Analysis and Machine Intelligence $25,713-724$.

Parzen, E., 1962. On estimation of a probability density function and mode. The Annals of Mathematical Statistics 33, 1065-1076.

Pejovic, T., Williams, V.A., Noland, R.B., Toumi, R., 2009. Factors affecting the frequency and severity of airport weather delays and the implications of climate change for future delays. Transportation Research Record: Journal of the Transportation Research Board , 97-106.

Perry, A.H., Symons, L.J., 1991. Highway Meteorology. University of Wales Swansea, Swansea, Wales, United Kingdom.

Shepard, F., 1996. Reduced Visibility Due to Fog on the Highway. 228.

Tan, R.T., 2008. Visibility in bad weather from a single image, in: IEEE Conference on Computer Vision and Pattern Recognition.

Tarel, J.P., Hautière, N., 2009. Fast visibility restoration from a single color or gray level image, in: IEEE International Conference on Computer Vision,
Kyoto, Japan.

Thach, T.Q., Wonga, C.M., , C.K.P., Chaua, Y., Chunga, Y.N., Oub, C.Q., Yanga, L., Hedleya, A.J., 2010. Daily visibility and mortality: Assessment of health benefits from improved visibility in Hong-Kong. Environmental Research 110, 617-623.

Torralba, A., Oliva, A., 2002. Depth estimation from image structure. IEEE Transactions on Pattern Analysis and Machine Intelligence 24, 1-13.

Xie, L., Chiu, A., Newsam, S., 2008. Estimating atmospheric visibility using general-purpose cameras, in: Bebis, G. (Ed.), International Symposium on Visual Computig, Part II, pp. 356-367. 